\title{
How Trust in Commercial Airline Pilots is Affected by Their Perceived Sociability: A Mediation Analysis
}

\author{
Stephen Rice, Scott R. Winter, and Russell Tokarski \\ Florida Institute of Technology
}

\begin{abstract}
Sociability relates to one's preference to interact with others or remain alone. The current study sought to determine how a pilot's perceived sociability would relate to consumers' trust ratings in their pilot using participants from India and the United States. Consumers were presented with one of two scenarios. In the control condition, the pilot was described as sociable, while in the other, the pilot was presented as unsociable. Participants were then asked to rate their trust in the pilot based off of these cues. In general, participants indicated that the pilot who was perceived as unsociable was less trusting compared to the pilot that was perceived as sociable. Americans tended to be more extreme in their trust ratings of the pilots than those participants from India. Finally, affect measures were also collected, and it was found that affect completely mediated the relationship between the sociable/unsociable conditions and trust ratings.
\end{abstract}

\section{Introduction}

Previous studies have examined how perceived sociability effects the public's perception of an airline pilot's mental health (Winter \& Rice, in press). The current study has taken this a step further by examining how the perceived sociability affects the public's trust in the pilot and predicts that affect will mediate the relationship between sociability and trust. The literature review will establish the link between a pilot's sociability and how it affects the trust of the pilot.

\section{Sociability}

Cheek and Buss (1981) defined sociability as "a tendency to affiliate with others and to prefer being with others to remaining alone" (p. 330). A person's sociability has been divided into two types: introvert or extrovert (Winter \& Rice, in press). Introverts are those who prefer to avoid social situations, while extroverts are usually thought of as outgoing and personable. A person may tend toward one type or the other, dependent on the situation or circumstance. Based on the outward cues of sociability that one purveys, others will form a perception of that person based on a mental model that is biased by stigma and affect. In a previous study, Rice and Winter (in press) noted that a pilot's perceived sociability led passengers to believe the pilot had some type of psychological disorder. This raises the question that if the same behavior were perceived in the person, but that person was not the pilot of the passenger's airplane; would it have led to the perception of a psychological disorder? At the same time, as Caldwell (2012) has explained, an individual's sociability has been associated with the levels of the neuropeptides; oxytocin 
and vasopressin, produced in the hypothalamus, and it could indicate a psychological disorder.

Fiske (1993) has posed that people tend to overreact to perceived negative information about a person. This is supported by Taylor's (1991) mobilization-minimization hypothesis. This poses that people respond to negative perceptions by first mobilizing psychologically, cognitively, emotionally, and socially, then by minimizing this internal stimuli. As far back as De Laguna (1919), it was recognized that perception and emotion are separate constructs, and that perceptual cues (perceptual qualities) are what spark emotional response (affective qualities). Now that it is established that perceptions are influenced by emotions, or affect, it will be necessary to establish what affect is and how it influences trust.

\section{Affect and Stigmas}

Recent research on affect has focused on its influence on decision-making (Bodenhausen, 1993; Bower, 1991; Clore, Schwarz, \& Conway, 1994; Forgas, 1995; Loewenstein, 1996; Schwarz \& Clore, 1996; Zajonc, 1998). It has been suggested that emotions assist in the decision making process when multiple streams of information require immediate processing simultaneously, and when there is the need for coordination of psychological, behavioral, and experiential responses (Frijda, 1986; Levenson, 1994; Oatley \& Johnson-Laird, 1996). It has even been suggested that cognitive processes may be interrupted by emotions during events that require deliberation, especially when those emotions are directing attention, memory, and judgment (Johnson-Laird \& Oatley, 1992; Lazarus, 1991; Schwarz, 1990; Simon, 1967; Tooby \& Cosmides, 1990). It has also been suggested that because of this, certain social stigmas may be the result of negative emotions (Winter \& Rice, in press). It seems that stigmas and affect are heavily relied upon during quick decision making when limited information may be available. The affect heuristic is highly influenced by strong emotion, with less reliance on the cognitive process (Alhakami \& Slovic, 1994). It has also been found that the affect heuristic has an inverse relationship with time pressure and emotional response (Finucane, Alhakami, Slovic, \& Johnson, 2000; Lowenstein, Weber, Hsee, \& Welch, 2001). Because of this research it could be suggested that affect has an impact on a person's perceptions of others, which could have an effect on their trust of that person.

Stigmas are defined as the prejudices that may be held against another because they are a part of or perceived to be a part of a specific group (Crocker, Major, \& Steele, 1998). Usually stigmas are associated with negative emotional reactions. They have been found to be associated with age, sexual orientation, gender, obesity, ethnic background, and physical or mental disabilities (Crocker, Major, \& Steele, 1998; Link \& Phelan, 2001; Mahjan et al., 2008). These stigmas may cause additional barriers for those afflicted with them, such as, social, economic or interpersonal (Crocker \& Major, 1989; Crocker, Voekle, Testa \& Major, 1991; Jones et al., 1984). Mental illness was one such stigma recently explored in another study. It was found that airline pilots who were perceived to be antisocial were labeled with the stigma of mental illness (Winter \& Rice, in press). Because of this stigma, it is theorized that pilots who are perceived as being less social than others will not be trusted. 


\section{Trust}

Trust has been defined in many ways, but the most effective definition for the current study is the ability to predict and rely on another's behavior (Deutsch, 1958; Eckel \& Wilson, 2004; Ergeneli, Saglam, \& Metin, 2007). If a person is able to rely on another's behavior, it means they have a significant amount of trust in that person. The perception is that if the person has trust in another and that trust is not fulfilled, then they are worse off than if they would not have trusted (Deutsch, 1958). Herein the basis for this research: if a passenger trusts a pilot to fly the aircraft and get them to their destination safely and the pilot fails to complete this task, the passenger would surely be worse off than if they would not have trusted in the first place.

There have been two forms of trust identified by McAllister (1995), cognitive and affect-based. Trust is cognition based because we choose who we trust and we base that decision on viable reasons (Lewis \& Weigert, 1985). Affect controls trust through emotional bonds between individuals (Lewis \& Weigert, 1985). If we do not trust another, we tend to hold that person in suspicion (Deutsch, 1958). For emotional or cognitive reasons, this person did not gain our trust, so we label them with the stigma of being untrustworthy.

Recent studies on trust and trust theory have centered on trust in automation, formulating the theory that the more failures in an automated system, the less a person trusted the system. It is believed that this can carry over to the human/human interaction systems as well. If a person is perceived to be unreliable, then the trust in them will wane (Winter, Rice, Reid, \& Mehta, 2015). Previous studies evaluated trust as an organizational behavior. In these studies, it was found that trust affects employee empowerment and managerial trust, as well as the trust between individual employees or groups within the organization (Ergeneli, Saglam, \& Metin, 2007). Mishra and Spreitzer (1998) have indicated that trust is dependent on one's emotional belief that the other party is reliable, competent, open, and concerned. This statement falls into line with McAllister's (1995) cognitive side of trust. If an individual or group provides evidence of being unreliable, incompetent, not open, or unconcerned about the other, they will be labeled as untrustworthy, causing an emotional response. This emotional response will provoke a negative stigma to be placed on that person.

In a previous study, it was found that social stigmas had an effect on a person's trust in an airline pilot, based on the pilot's gender, age, physical structure, and ethnicity (Winter, Rice, \& Mehta, 2014). It has also been found that a person's trust is highly influenced by another's sexuality, or social status, suggesting that trust is highly affected by emotions. Through mediation analysis in these previous studies it was found that affect tends to have a mediating relationship between the condition and trust. Another study also found that persons in India and the United States felt that airline pilots who are perceived to be unsociable may be tagged with the stigma of mental illness (Winter \& Rice, in press). The stigma of mental illness is one of negativity. In both India and the U.S., persons stigmatized as being afflicted with mental illness are viewed as unstable and are limited in their duties 
or responsibilities (Stanhope, 2002; Corrigan, Markowitz, \& Watson, 2004), indicating a lack of trust in those individuals or bestowing upon them the stigma of untrustworthiness.

\section{Current Study}

The current study expands on previous research on pilot sociability (Winter \& Rice, in press). The researchers were specifically interested in how trust ratings would be affected by perceived sociability. Since participants in the earlier study viewed unsociable pilots as more likely to have a mental illness, there was desire to determine if consumers would have lower trust ratings of unsociable pilots as well. Finally, the research team sought to determine whether affect was a mediating variable between sociability and trust. The study consisted of individuals from both India and the United States to inspect for any cultural differences. Affect measures were collected to determine if affect had any mediating effect on the relationship between sociability and trust. The authors predicted the following:

$\mathrm{H}_{1}$ : Pilots who are viewed as unsociable will also be viewed as less trustworthy by participants.

$\mathrm{H}_{2}$ : Americans will be more extreme in their Affect and Trust ratings compared to Indian participants. There is some evidence of this in previous studies (Rice et al., 2014; Remy, Winter, \& Rice, 2014; Winter, Rice, \& Mehta, 2014).

$\mathrm{H}_{3}$ : Affect will act as a mediator, at least partially, between sociability and trust. There is some evidence of this in the mental health literature (Richardson \& Rice, in press; Rice, Richardson, \& Kraemer, in press).

\section{Methods}

\section{Participants}

Participants for the study were recruited from India and the United States via a convenience sample from Amazon's ${ }^{\circledR}$ Mechanical Turk ${ }^{\circledR}$ (MTurk). MTurk is an online repository of participants from around the globe that complete human intelligence tasks (HITs) for monetary compensation. Previous research (Buhrmester, Kwang, \& Gosling, 2011; Germine et al., 2012) has demonstrated that data collected via MTurk is just as reliable as data collected from traditional laboratory settings.

Three hundred and nine participants (127 females) from India completed the study. The mean age for participants was $31.56(S D=9.63)$. Three hundred and seventeen participants (135 females) from the United States completed the study. The mean age was 31.14 $(S D=10.30)$.

\section{Materials, Stimuli, and Design}

An electronic consent form was completed by participants to verify they were at least 18 years old. In the control condition, participants reviewed the following scenario: Imagine that you are on a commercial airline flight from one major city to another. As you are preparing to board, you overhear one of the flight attendants telling the other that the pilot has recently been acting like his usual cheerful self. He has been communicative with his crew and friends. He has posted positive messages on social media and Facebook in 
the past month. He has been pleasant to his co-pilots." In the experimental condition, the following scenario was presented: "Imagine that you are on a commercial airline flight from one major city to another. As you are preparing to board, you overhear one of the flight attendants telling the other that the pilot has recently been acting strange and not like his usual self. He has lost his temper twice in the past two weeks. He has not been very communicative with his crew or friends. He has avoided social media. He has not posted to Facebook in the past month. He has been rude to his co-pilot on several occasions."

Participants from both conditions were asked three affect questions on a 7-point Likerttype scale to rate how the respective scenarios made them feel. These responses ranged from extremely negative/unfavorable/bad (-3) to extremely positive/favorable/good (+3), with a neutral option of zero. The gathering of these affect measures followed a similar procedure as completed in previous research (Rice, Richardson, \& Kraemer, in press; Winter, Rice, \& Mehta, 2014). Appendix B shows the affect measures.

Participants in both conditions were then asked to rate their trust in the pilot and how trustworthy they thought he/she would be based on the information provided in the scenario. The study used an instrument called the Trust in Commercial Airline Pilots (TCAP) scale, which is provided in Appendix A. This instrument was validated by Rice, Mehta, Winter, and Oyman (2015) and consists of 5 items measured on a five-point Likert scale from strongly disagree (-2) to strongly agree $(+2)$, with a neutral option of zero. Finally, demographic information was collected from participants before completion of the study.

The study used a three-way between-participants design. Country of Origin, Gender, and Sociability were the independent variables. The dependent variables for the study were affect (mediator variable) and trust.

\section{Results}

Factorial Analyses. First, a Cronbach's Alpha was conducted on the three Affect questions. Values ranged from .88 to .96 , indicating high internal consistency. Therefore, these data were combined into one measure for analysis purposes. A three-way ANOVA was completed with Country, Gender, and Sociability as between-participant variables. For the Affect dependent variable, there was a significant main effect for Sociability, $F(1,618)$ $=893.83, p<0.001, n p^{2}=.59$, and there was a significant interaction between Sociability and Country, $F(1,618)=30.95, p<0.001, n p^{2}=.05$. These data, shown in Figure 1, suggest that pilot sociability impacts Affect ratings of participants, and that participants from the United States produced more extreme responses in both conditions compared to participants from India $(\mathrm{p}<0.05)$. 


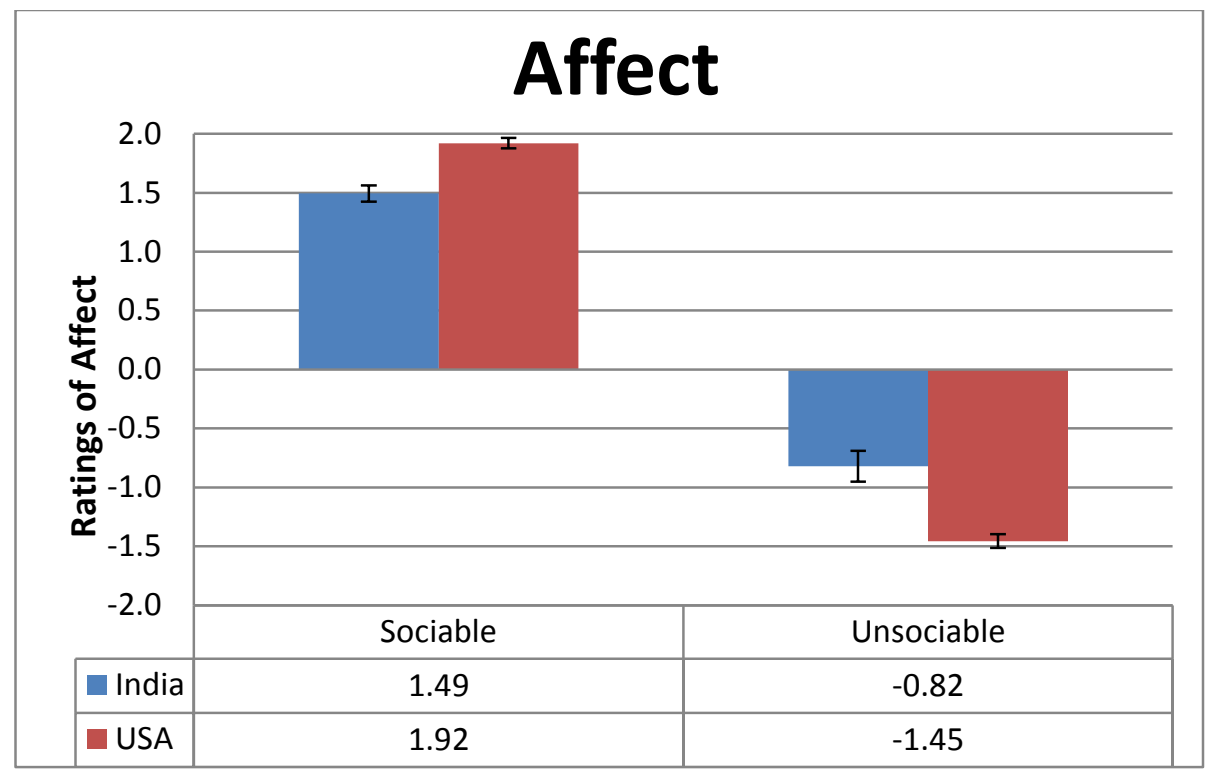

Figure 1. Affect data from the study (SE bars included).

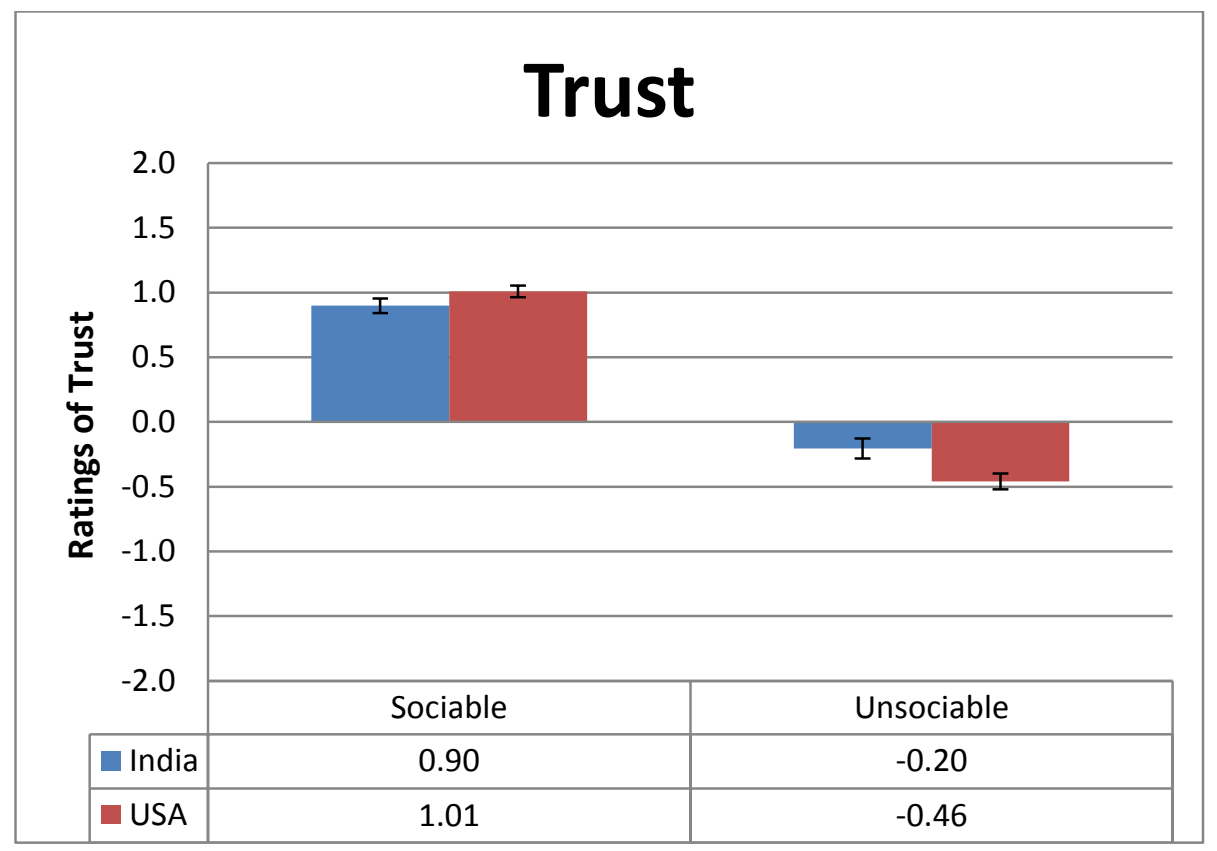

Figure 2. Trust data from the study (SE bars included).

The data for trust ratings were also subjected to a Cronbach's Alpha analysis. Values ranged from .88 to .92 , indicating high internal consistency. Therefore, the trust measures were merged into one dependent variable for analysis. A three-way ANOVA, with Country, Gender, and Sociability as between-participant variables, indicated a significant main effect for Sociability, $F(1,618)=254.94, p<0.001, n p^{2}=.41$, along with a significant interaction between Sociability and Country, $F(1,618)=8.65, p<0.01, n p^{2}=.01$. As 
Figure 2 shows, Americans tended to be more extreme in their responses; however, this was only statistically significant for the Unsociable condition $(p<0.01)$.

Mediation Analyses. Figure 3 provides a graphical depiction of the mediation analysis performed on the data for American participants. To complete a mediation analysis, a correlation must first exist between the initial variable (sociable or unsociable) and the outcome variable (trust). This relationship was shown to be significant, $r=.739, p<.001$. The standardized path coefficients were: condition to affect $(.901, p<.001)$; affect to trust (.930, $p<.001)$; condition to trust controlling for affect $(-.100, p=.157)$. These data suggests that affect completely mediated the relationship between condition and trust for American participants.

US

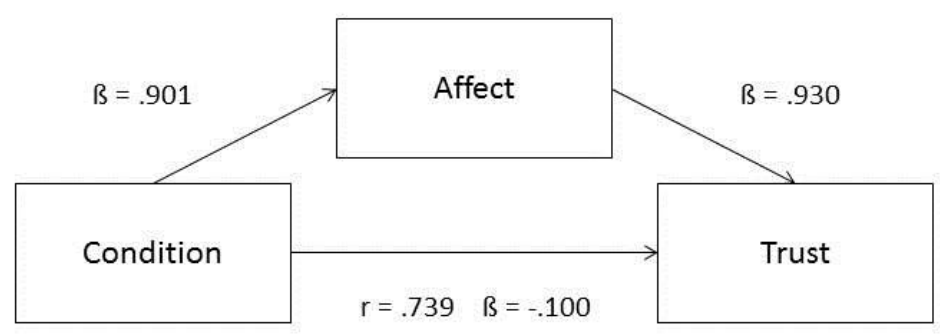

Figure 3. Path analysis for American participants.

Figure 4 depicts the mediation analyses for Indian participants. Before completing the mediation analysis, a significant correlation was shown to exist between the initial variable (sociable and unsociable) and outcome variable (trust), $r=.544, p<.001$. The standardized path coefficients were: condition to affect $(.620, p<.001)$; affect to trust $(.787, p<.001)$; and condition to trust controlling for affect $(.056, p=.177)$. These data suggest that affect completely mediated the relationship between condition and trust for Indian participants.

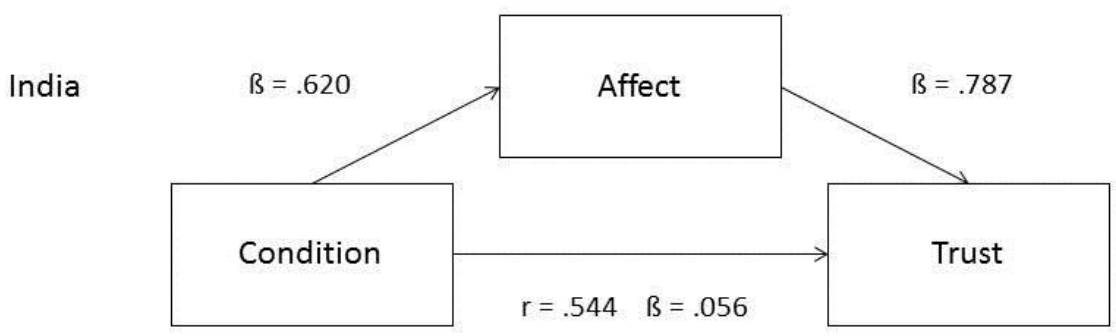

Figure 4. Path analysis for Indian participants. 


\section{General Discussion}

This study determines how the perceived sociability of a pilot would influence the trust ratings of consumers. Prior research (Winter \& Rice, in press) has demonstrated that sociability cues toward a pilot affect participant's opinion as to whether that pilot is suffering or likely to be suffering from a mental illness. Based on this finding, the authors wanted to determine how trust ratings would be affected by perceived sociability.

The first hypothesis predicted that pilots who were viewed as more sociable would also be viewed as more trustworthy than those viewed as less sociable. The data supported this hypothesis across both Indian and American participants. Pilots who were viewed as unsociable were identified as less trusting than those who were identified as sociable. As identified by Caldwell (2012), an individual's sociability level has been linked to various mental parameters and may be indicative of a psychological disorder. Additionally, Fiske (1993) has highlighted that persons tend to overreact toward negative information when received about another person. Therefore, it is not unreasonable to interpret participants' drops in trust ratings upon perceiving the pilot to be unsociable. Stigmas held towards those that are less sociable may also explain the drop in trust ratings. Crocker, Major, and Steele (1998) describe how prejudices are held against another because they are or are perceived to be part of a specific group, and these stigmas tend to be negative. Finally, people tend to trust most when they are able to predict the behavior and/or actions of another person (Deutsch, 1958; Eckel \& Wilson, 2004; Ergeneli, Saglam, \& Metin, 2007). If perceived as unsociable, it is plausible that participants may have felt the pilot was less predictable and therefore were willing to trust that individual less when compared to the sociable pilot.

The second hypothesis predicted that American participants would be more extreme in their ratings of trust than Indian participants as has been witnessed in previous studies (Rice et al. 2014; Remy, Winter, \& Rice, 2014; Winter, Rice, \& Mehta, 2014). The findings of the study, in general, support this hypothesis. When reviewing measures of affect and trust, American participants had higher ratings for the sociable pilot and lower ratings of the unsociable pilot than the Indian participants. However, while the trust rating for the sociable pilot was higher for Americans, it was not significantly different compared to the Indian participants. These findings, for the most part, are in agreement with the previously identified research. A possible explanation for these differences may be related to the specific cultures of each nationality. Americans tend to be more individualistic in their culture while Indians are more collectivist (Markus \& Kitayama, 1991). Those from individualistic cultures are less likely to trust without question. Meanwhile those from collectivist cultures view themselves in the context of the population as a whole, may be more likely to trust without question, and less likely to challenge authority (Markus \& Kitayama, 1991).

The third hypothesis predicted that Affect would act as a mediator, at least partially, between the sociability and trust ratings. Basis for this hypothesis was grounded in prior research from the mental health field (Richardson \& Rice, in press; Rice, Richardson, \& Kraemer, in press). The data supported this finding and Affect completely mediated the relationship between sociability and trust for both Indian and American participants. These 
findings suggest that the views held toward the trust ratings of a pilot perceived as sociable or unsociable are highly influenced by emotions, which can also affect judgment. This finding is similar to earlier studies that have shown Affect to mediate relationships toward trust in pilots (Remy, Winter, \& Rice, 2014; Winter, Rice, \& Mehta, 2014). Literature has shown that Affect plays a role in the decision-making process of individuals, especially when those decisions must be made in short periods of time. Additionally, the affect heuristic is highly influenced by strong emotions. It is possible that when participants were completing the study and had to quickly make a determination as to the trust of the pilot, emotional reactions heavily influenced the ratings.

\section{Practical Implications and Limitations}

It appears from the findings that participants were more trusting of a pilot perceived as more sociable. While pilots are employed to safely operate the aircraft, they also serve in the role of a company representative. Often times the flight crew are the most visible members of the airline. It is important for these persons to remember that their perceived sociability may have an effect on the consumer's overall experience during the flight, regardless of how well the flight goes. Further research should be completed to determine if similar findings are reported when the type of airline personnel is manipulated, such as flight attendants or gate agents. Additionally from an airline marketing perspective, portraying crew members in a sociable light may assist in creating trusting opinions of the flying public toward those individuals and the airline.

Certain limitations exist in the current study. First, the study is limited to those types of participants that complete online human intelligence tasks. These individuals tend to be current with technology, and a younger demographic. Therefore, generalizations of the findings must be limited to this population, which may not be representative of the population as a whole. Further research can expand the sampling technique to verify the results of this study and produce results with greater generalizability. Additionally, only two nationalities were reviewed in the current study, Indians and Americans. Further research could expand to various nationalities to see how other cultural aspects may influence the study's findings. Finally, participants may have been primed by the wording of the scenario for the pilot depicted as unsociable.

\section{Conclusions}

The findings of this study are similar to, and expand upon, previous studies completed in this field of research. When a pilot is perceived as sociable, participants tended to trust that pilot more than one that was perceived as unsociable. Americans, in general, tended to be more extreme in their trust ratings than Indian participants, which may be attributed toward the cultural differences between the two groups. Finally, affect completely mediated the relationship between the condition and trust ratings which signifies that participant responses were heavily influenced by emotions as opposed to cognition. 


\section{References}

Alhakami, A. S., \& Slovic, P. (1994). A psychological study of the inverse relationship between perceived risk and perceived benefit. Risk Analysis, 14, 1085-1096.

Bodenhausen, G. V. (1993). Emotions, arousal, and stereotypic judgments: A heuristic model of affect and stereotyping. In D .M. Mackie \& D. L. Hamilton (Eds.), Affect, cognition, and stereotyping: Interactive processes in group perception (pp. 13-37). San Diego, CA: Academic Press.

Bower, G. H. (1991). Mood congruity of social judgment. In J. Forgas (Ed.), Emotion and social judgment (pp. 31-54). Oxford, UK: Pergamon.

Buhrmester, M., Kwang, T., \& Gosling, S. D. (2011). Amazon's Mechanical Turk: A new source of inexpensive, yet high-quality data? Perspectives on Psychological Science, 6(3), 3-5.

Caldwell, H. K. (2012). Neurobiology of sociability. In Lopez-Larrea, C. (Ed.), Sensing in Nature. (pp. 187-205). New York, NY: Springer US.

Cheek, J. M. \& Buss, A. H. (1981). Shyness and sociability. Journal of Personality and Social Psychology, 41(2), 330-339.

Clore, G. L., Schwarz, N., \& Conway, M. (1994). Cognitive causes and consequences of emotion. In R. S. Wyer, Jr. \& T. K. Srull (Eds.), Handbook of social cognition (2nd ed., pp. 323-417). Hillsdale, NJ: Erlbaum.

Corrigan, P. W., Markowitz, F. E., \& Watson, A. C. (2004). Structural levels of mental illness stigma and discrimination. Schizophrenia Bulletin, 481-491.

Crocker, J., \& Major, B. (1989). Social stigma and self-esteem: The self-protective properties of stigma. Psychological Review, 26, 608-630.

Crocker, J., Major, B., \& Steele, C. (1998). Social stigma. In D. T. Gilbert, \& S. T. Fiske (Eds.), The handbook of social psychology (pp. 504-553). New York: McGraw-Hill.

Crocker, J., Voelkl, K., Testa, M., \& Major, B. (1991). Social stigma: The affective consequences of attributional ambiguity. Journal of Personality and Social Psychology, 60, 218-228.

De Laguna, G. A. (1919). Emotion and perception from the behaviorist standpoint. Psychology Review. 26(6). 409-427.

Deutsch, M. (1958). Trust and suspicion. The Journal of Conflict Resolution, 2, 265-279. 
Eckel, C. C. \& Wilson, R. K. (2004). Is trust a risky decision? Journal of Economic Behavior \& Organization, 55, 447-465.

Ergeneli, A., Saglam, G., \& Metin, S. (2007). Psychological empowerment and its relationship to trust in immediate managers. Journal of Business Research, 60, 41-49.

Finucane, M. L., Alhakami, A., Slovic, P., \& Johnson, S. M. (2000). The affect heuristic in judgments of risks and benefits. Journal of Behavioral Decision Making, 13, 1-17.

Fiske, S. T. (1993). Social cognition and social perception. Annual Review of Psychology. 44. 155-194.

Forgas, J. P. (1995). Mood and judgment: The affect infusion model (AIM). Psychological Bulletin, 117, 39-66.

Frijda, N. H. (1986). The emotions. Cambridge, UK: Cambridge University Press.

Germine, L., Nakayama, K., Duchaine, B.C., Chabris, C.F., Chatterjee, G., \& Wilmer, J.B. (2012) Is the web as good as the lab? Comparable performance from web and lab in cognitive/perceptual experiments. Psychonomic Bulletin \& Review, 19(5), 847-857.

Johnson-Laird, P. N., \& Oatley, K. (1992). Basic emotions, rationality, and folk theory. Cognition and Emotion, 6, 201-223.

Jones, E. E., Farina, A., Hastorf, A. H., Markus, H., Miller, D. T., \& Scott, R. A. (1984). The dimensions of stigma: The psychology of marked relationships. New York: $\mathrm{W} . \mathrm{H}$. Freeman Company.

Lazarus, R.S. (1991). Emotion and adaptation. New York: Oxford University Press.

Levenson, R. (1994). Human emotion: a functional view. In P. Ekman \& R. J. Davidson (Eds.), The nature of emotion (pp. 123-126). New York: Oxford University Press.

Lewis, J. D., \& Weigert, A. (1985). Trust as a social reality. Social Forces, 63, 967-985.

Link, B. G., \& Phelan, J. C. (2001). Conceptualizing stigma. Annual Review Sociology, 27, 363-385.

Loewenstein, G. (1996). Out of control: Visceral influences on behavior. Organizational Behavior and Human Decision Processes, 65, 272-292.

Loewenstein, G., Weber, E., Hsee, C., \& Welch, N. (2001). Risk as feelings. Psychological Bulletin, 127, 267-286. 
Mahjan, A. P., Sayles, J. N., Patel, V. A., Remien, R. H., Ortiz, D., Szekeres, G., et al. (2008). Stigma in the HIV/AIDS epidemic: A review of the literature and recommendations for the way forward. AIDS, 22, 67-79.

Markus, H. R. \& Kitayama, S. (1991). Culture and the self: Implications for cognition, emotion, and motivation. Psychological Review, 98(2): 224-253.

McAllister, Daniel J. (1995). Affect- and cognition-based trust as foundations for interpersonal cooperation in organizations. Academy of Management Journal, 38(1):24-59.

Mishra, A. K. \& Spreitzer, G. M. (1998). Explaining how survivors respond to downsizing: The role of trust, empowerment, justice, and work redesign. Academy of Management Review, 23(3), 568-588.

Oatley, K., \& Johnson-Laird, P. N. (1996). The communicative theory of emotions: Empirical tests, mental models, and implications for social interaction. In L. L. Martin \& A. Tesser (Eds.), Striving and feeling: Interactions among goals, affect, and self-regulation. Hillsdale, NJ: Erlbaum.

Remy, B., Winter, S. R., \& Rice, S. (2014, April). American aviation consumer's trust in pilots. Presentation at the $7^{\text {th }}$ annual Human Factors and Applied Psychology Student Conference, Daytona Beach, FL.

Rice, S., Kraemer, K., Winter, S. R., Mehta, R., Dunbar, V., Rosser, T. G., \& Moore, J. C. (2014). Passengers from India and the United States have differential opinions about autonomous auto-pilots for commercial flights. International Journal of Aviation, Aeronautics, and Aerospace, 1(1), 1-12.

Rice, S., Mehta, R., Winter, S.R., \& Oyman, K. (2015). A Trustworthiness of Commercial Airline Pilots (T-CAP) Scale for American Consumers. Journal of Aviation Technology and Engineering, 4(2), 55-63.

Rice, S., Richardson, J. \& Kraemer, K. (in press). The emotional mediation of distrust of persons with a mental illness. International Journal of Mental Health.

Richardson, J., \& Rice, S. (in press). The role of affect and cognition in the public's judgments about the need for mental health treatment and willingness to help. International Journal of Mental Health.

Schwarz, N. (1990). Feelings as information: Informational and motivational functions of affective states. In E. T. Higgins \& R. M. Sorrentino (Eds.), Handbook of motivation and cognition: Foundation s of social behavior (Vol. 2, pp. 527-561). New York: Guilford Press. 
Schwarz, N., \& Clore, G. (1996). Feelings and phenomenal experiences. In E. T. Higgins \& A.W. Kruglanski (Eds.), Social psychology: Handbook of basic principles (pp. 433-465). New York: Guilford Press.

Simon, H. A. (1967). Motivational and emotional controls of cognition. Psychological Review, 74, 29-39.

Stanhope, V. (2002). Culture, control, and family involvement: A comparison of psychosocial rehabilitation in India and the United States. Psychiatric Rehabilitation Journal, 273-280.

Taylor, S. E. (1991). Asymetrical effects of positive and negative events: The mobilization-minimization hypothesis. Psychological Bulletin. 110(1). 67-85.

Tooby, J., \& Cosmides, L. (1990). The past explains the present: Emotional adaptations and the structure of ancestral environments. Ethology and Sociobiology, 11, 375-424.

Winter, S. R. \& Rice. S. (In press). Trust of Commercial Pilots May Be Related to Sociability: An Affective Perspective. Aviation Psychology and Applied Human Factors.

Winter, S. R., Rice, S., \& Mehta, R. (2014). Aviation consumers' trust in pilots: A cognitive or emotional function. International Journal of Aviation, Aeronautics, and Aerospace, 1(1), 1-18.

Winter, S. R., Rice, S., Reid, K. M., \& Mehta, R. (2015). Consistency of aviation students when taking the private pilot knowledge exam. Journal of Aviation/Aerospace Educational Research, 24(2), 21-38.

Zajonc, R. (1998). Emotions. In D. Gilbert, S. Fiske, \& G. Lindzey (Eds.), The handbook of social psychology (Vol. 1, pp. 591-632). New York: Oxford University Press. 


\section{Appendix A}

\section{Trust of Commercial Airline Pilots Scale}

Please respond how strongly you agree or disagree with the following statements.

1. The pilot is dependable.

Strongly Disagree Disagree Neutral Agree Strongly Agree

2. The pilot is reliable.

Strongly Disagree Disagree Neutral Agree Strongly Agree

3. The pilot is responsible.

Strongly Disagree Disagree Neutral Agree Strongly Agree

4. The pilot is safe.

Strongly Disagree Disagree Neutral Agree Strongly Agree

5. The pilot is trustworthy.

Strongly Disagree Disagree Neutral Agree Strongly Agree




\section{Appendix B}

\section{Affect Measures}

Please respond to how the scenario makes you feel:

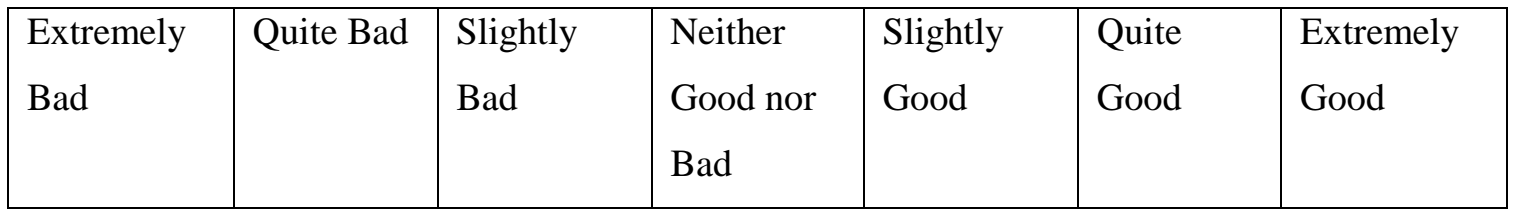

Please respond to how the scenario makes you feel:

\begin{tabular}{|l|l|l|l|l|l|l|}
\hline Extremely & Quite & Slightly & Neither & Slightly & Quite & Extremely \\
Unfavorable & Unfavorable & Unfavorable & $\begin{array}{l}\text { Unfavorable } \\
\text { nor } \\
\text { Favorable }\end{array}$ & Favorable & Favorable & Favorable \\
& & & & & \\
\hline
\end{tabular}

Please respond to how the scenario makes you feel:

\begin{tabular}{|l|l|l|l|l|l|l|}
\hline Extremely & Quite & Slightly & Neither & Slightly & Quite & Extremely \\
Negative & Negative & Negative & $\begin{array}{l}\text { Negative } \\
\text { nor } \\
\text { Positive }\end{array}$ & Positive & Positive & Positive \\
& & & & \\
\hline
\end{tabular}

